\title{
Author Correction to: Dusukasi-The Heart That Cries: An Idiom of Mental Distress Among Perinatal Women in Rural Mali
}

\author{
Molly E. Lasater ${ }^{1} \cdot$ Madeleine Beebe $^{1}$. \\ Nicole E. Warren ${ }^{2}$. Fatoumata Souko ${ }^{3}$. \\ Mariam Keita ${ }^{3} \cdot$ Sarah M. Murray ${ }^{4}$. \\ Judith K. Bass ${ }^{4} \cdot$ Pamela J. Surkan ${ }^{1}$. \\ Peter J. Winch ${ }^{1}$
}

Published online: 2 June 2018

(C) The Author(s) 2018

\section{Correction to: Cult Med Psychiatry https://doi.org/10.1007/s11013-018-9579-6}

The original version of this article unfortunately contained a mistake in the author name.

The co-author name should be Sarah M. Murray instead of Sarah E. Murray.

The original article has been corrected.

The original article can be found online at https://doi.org/10.1007/s11013-018-9579-6.

$\triangle$ Molly E. Lasater

Mlasater@jhu.edu

1 Social and Behavioral Interventions Program, Department of International Health, Johns

Hopkins Bloomberg School of Public Health, 615 North Wolfe Street, Baltimore,

MD 21205-2103, USA

2 School of Nursing, Johns Hopkins Bloomberg School of Public Health, Baltimore, MD, USA

3 Department of Public Health, University of Sciences, Techniques \& Technologies of Bamako, Bamako, Mali

4 Department of Mental Health, Johns Hopkins Bloomberg School of Public Health, Baltimore, MD, USA 